\title{
Le développement des stratégies de régulation affective au cours de l'âge adulte
}

\author{
Sarah Le Vigouroux ${ }^{*}$, Bruno Dauvier ${ }^{1}$, Anne Congard $^{1}$, \\ Jean-Luc Kop ${ }^{2}$, Jean-Baptiste Pavani ${ }^{1}$ et Pierre-Yves Gilles ${ }^{1}$ \\ ${ }^{1}$ Aix-Marseille Université, Centre PsyCLE, Aix-en-Provence, France \\ ${ }^{2}$ Université de Lorraine, InterPsy, Nancy, France
}

\begin{abstract}
RÉSUMÉ
Alors que l'avancée en âge est associée à une baisse de l'efficacité de certains processus cognitifs et physiques (Carstensen, Gross, \& Fung, 1998), de nombreuses études font état d'une augmentation du bien-être général chez les personnes âgées (par ex. Urry \& Gross, 2010). L'acquisition d'une forme d'expertise des stratégies dans la régulation des affects chez l'adulte est une explication parfois proposée dans la littérature pour en rendre compte. Dans le but d'étudier, dans une perspective vie entière, l'évolution avec l'âge des processus de sélection et de l'efficacité des stratégies de régulation affective, nous avons utilisé un protocole de recueil de données en situation de vie quotidienne sur un échantillon de 172 personnes de 18 à 70 ans. Nos résultats indiquent un phénomène de sélection de certaines stratégies de régulation affective qui sont privilégiées avec l'avancée en âge : le coping centré sur le problème et la réévaluation cognitive. Ces deux stratégies deviennent également de plus en plus efficaces pour favoriser les affects positifs et diminuer les affects négatifs.
\end{abstract}

The development of strategies affective regulation during adulthood

ABSTRACT

While aging is associated with a reduction in the effectiveness of some cognitive and physical processes (Carstensen, Gross \& Fung, 1998), many studies have reported an increase in general well-being in the elderly (e.g. Urry \& Gross, 2010). The acquisition of skills in affect regulation during adulthood is sometimes suggested in the literature as an explanation. In order to study the evolution with age of the selection process and the effectiveness of strategies for affective regulation in a lifespan perspective, we used an experience sampling

*Correspondance : Sarah Le Vigouroux, Aix-Marseille Université, Centre PsyClé, 29, avenue Robert Schuman, 13621 Aix en Provence Cedex 1. E-mail : sarah.le.vigouroux@univ-amu.fr

Remerciements. Nous tenons à remercier Élodie Chabert et Guillaume Beuriot, qui ont contribué au recueil de données, ainsi que tous les participants de cette étude. 
methodology on a sample 172 people aged from 18 to 70 years. Results indicate a process of selection of some affective regulation strategies that are favoured with age : the problem focused coping and cognitive reappraisal. Both strategies are also becoming increasingly effective in promoting positive affect and lower negative affect.

\section{INTRODUCTION}

L'avancée en âge est marquée par des diminutions des compétences physiques, souvent cognitives et parfois sociales (Carstensen et al., 1998). Pourtant, de nombreuses études font état d'une augmentation du bien-être général chez les personnes âgées (Urry \& Gross, 2010 ; Cacioppo, Hawkley, Kalil, Hughes, Waite, \& Thisted, 2008 ; Guillaume, Eustache, \& Desgrange, 2009). Comment les personnes plus âgées parviennent-elles à se sentir mieux, alors qu'elles peuvent vivre des événements plus négatifs en moyenne ? Cela s'expliquerait par le développement au cours de l'âge adulte d'une forme d'expertise dans la régulation des affects. Les affects, même s'ils ont une fonction adaptative qui permet à la personne de réagir de manière appropriée face aux situations qu'elle rencontre (Piolat \& Bannour, 2008), doivent être régulés lorsqu'ils deviennent trop intenses. Il s'avère souvent nécessaire de tenter volontairement d'agir sur eux afin de retrouver une certaine stabilité de l'état affectif et de maintenir une forme d'homéostasie. Ceci est rendu possible grâce, entre autres, à l'utilisation de stratégies de régulation permettant de rapprocher l'état affectif d'un niveau moyen propre à chaque individu (Kuppens, 2010). Gross (1998) définit le phénomène de régulation comme les processus que les individus emploient pour influencer les affects qu'ils ressentent, au moment où ils les ont et comment ils les éprouvent et les expriment. La régulation requiert la mobilisation d'un ensemble de stratégies d'ajustement dans le but d'adapter le comportement affectif au contexte et aux normes socioculturelles et/ou de faciliter l'atteinte des buts et des besoins (Gross, 1998 ; Gross, Richards, \& John, 2006).

Les affects seraient les reflets des événements perçus au travers du filtre des processus de régulation. Le lien entre l'âge et la régulation pourrait s'expliquer par trois principes : 1 . une meilleure sélection et utilisation des stratégies ciblées comme efficaces (modèle de Sélectivité Socioémotionnelle : Carstensen, Gross, \& Fung, 1998 ; Carstensen, 1995) ; 2. une meilleure optimisation des ressources pour les mettre en place avec un coût cognitif moindre (modèle de la Sélection-OptimisationCompensation en Régulation Émotionnelle : Urry \& Gross, 2010) ; 3. 
une meilleure capacité à prévenir la survenue d'un épisode affectif négatif par l'utilisation de stratégies proactives (telle que dans la résolution de problème : Blanchard-Fields, 2007). Dans cette étude, la régulation affective est vue comme un système dynamique (Kuppens, 2010), dont la fonction est de maintenir un état d'homéostasie. L'objectif est alors d'étudier ces processus de sélection, d'optimisation et de prévision d'affects négatifs, s'inscrivant dans une dynamique temporelle composée de variations au cours d'une même journée.

\subsection{Les stratégies de régulation}

Dans cette étude, nous avons retenu six stratégies de régulation parmi les plus étudiées dans la littérature. Certaines sont clairement identifiées comme étant plus efficaces que d'autres. Les trois premières viennent des travaux sur les stratégies de coping. Le coping centré sur le problème vise à modifier la situation dans le but d'en réduire les exigences et/ou d'augmenter ses propres ressources pour mieux faire face au problème. Il peut se faire en réaction ou en prévention (c'est-à-dire de façon proactive) de situations entraînant des affects négatifs. C'est la stratégie la plus exigeante en ressources personnelles (cognitives, motivationnelles...), mais généralement la plus efficace dans la diminution des affects négatifs (Lazarus \& Folkman, 1984). Le coping centré sur le soutien social correspond à la recherche de l'aide qu'autrui peut apporter. Il comporte trois facettes : le réseau social (le nombre de relations, la fréquence des contacts et l'intensité des liens), le soutien social perçu (l'impact subjectif de l'aide reçue) et le soutien social reçu (l'aide effective ; Boujut \& Bruchon-Schweitzer, 2007). Cette stratégie est considérée comme relativement efficace par la prolongation des affects positifs et la diminution des affects négatifs. Généralement elle est utilisée de façon réactive. L'évitement a pour but de faire diminuer les affects négatifs par des actions de détournement/fuite qui amènent l'individu à entrer dans des activités autres que la confrontation à la situation responsable de ces affects. Cette stratégie est généralement considérée comme peu efficace dans les études par questionnaires. Cependant en situation expérimentale, la non-confrontation à certains événements peut se révéler efficace, surtout si la personne a peu de contrôle sur la situation (Boujut \& Bruchon-Schweitzer, 2007).

La réévaluation cognitive et la suppression expressive sont deux stratégies décrites par Gross et Thompson (2007). La réévaluation cognitive consiste en un changement d'interprétation de la séquence affective et de ses causes, dont l'effet serait une modification subjective de l'affect - négatif 
ou positif - et incidemment du bien-être psychologique. Elle vise à sélectionner certains aspects d'une situation, lors de l'anticipation de ses conséquences ou a posteriori, afin de moduler les affects générés, par exemple la tristesse ressentie. La réévaluation cognitive est une stratégie qui peut être utilisée aussi bien de façon réactive en reconsidérant un événement passé, que proactive en relativisant ce qui s'est passé ou ce qui pourrait se passer (Garnefski, van den Kommer, Kraaii, Teerds, Legerstee, \& Onstein, 2002), mais aussi en cherchant à mettre en avant les aspects positifs des conséquences éventuelles. Considérer qu'un échec - potentiel ou passé - est aussi une occasion d'apprentissage, constitue une forme de réévaluation cognitive. Cette stratégie est considérée comme étant très efficace. Elle est notamment utilisée dans différentes thérapies. Son utilisation est généralement associée à des affects positifs plus fréquents et à moins d'affects négatifs (Gross \& John, 2003). Une autre stratégie observée, la suppression expressive, correspond à l'inhibition de l'expression des affects dans le but de ne pas rendre visible son propre état affectif. Il s'agit alors de modifier la réponse comportementale affective qu'elle soit positive ou négative, après qu'elle ait été engendrée (Gross \& John, 2003) sans pour autant venir influencer l'état affectif en lui-même.

La rumination est également une stratégie de régulation, bien qu'elle soit généralement considérée comme moins efficace que les précédentes (Luminet, 2002). Elle est utilisée dans un objectif de recherche de sens après le vécu d'une situation. À court terme, elle permet généralement une réponse adaptative aux situations affectives par assimilation progressive de la réalité nouvelle aux schémas existants (Luminet, 2002). À plus long terme, elle peut engendrer un maintien prolongé dans un état affectif à valence négative si elle n'aboutit pas sur une forme de réévaluation cognitive. Son utilisation est généralement associée à un état de tristesse plus ou moins intense.

\subsection{La structure dimensionnelle des affects}

Si le terme émotion renvoie à des réactions brèves et intenses (Lazarus, 1991), les affects sont considérés comme des états qui peuvent être ressentis de manière plus durable (Watson \& Clark, 1984). L'état affectif renvoie à la composante expérientielle des émotions, c'est-à-dire le ressenti subjectif dont l'individu a conscience. Dans le cadre de l'approche dimensionnelle proposée par des auteurs tels que Russell (1980) ou Watson (1988), les affects sont identifiés et leur structure décrite sur la base des corrélations entre les réponses données par les individus lorsqu'ils sont interrogés 
sur leurs ressentis (Russell \& Carroll, 1999 ; Watson \& Tellegen, 1999). En 1980, Russell propose un modèle circomplexe des affects obtenu par analyse factorielle. Dans le modèle de Russell et Barrett (1999), les affects apparaissent disposés en cercle dans un espace bidimensionnel selon deux dimensions orthogonales bipolaires : la valence (plaisante-déplaisante) et l'activation (ou éveil, actif-inactif). Cette conception est devenue la représentation structurale la plus utilisée dans les travaux sur les différences individuelles (Wilt, Funkhouser, \& Revelle, 2011). Dans sa version la plus aboutie (Yik, Russell, \& Steiger, 2011), le circomplexe est composé de douze cadrans. Dans cette étude les notions d'Affects Positifs (AP) et d'Affects Négatifs (AN) renvoient à la conception de la valence (plaisante-déplaisante) telle que définie par Russell.

Cependant le modèle circomplexe des affects ne prend pas bien en compte la possibilité qu'une personne puisse ressentir simultanément de l'affectivité positive et de l'affectivité négative ou aucune des deux. Pourtant les états affectifs neutres en termes de valence et en termes d'activation paraissent vraisemblables. De plus, Russell et Carroll (1999) ont constaté que certains de leurs participants $(11 \%)$ se sont sentis heureux et tristes en même temps et Larsen, McGraw et Cacioppo (2001) ont démontré qu'il est possible de ressentir de la joie et de la tristesse simultanément. Le modèle d'espace d'évaluation de Cacioppo et Berntson (1994) sépare Affects Positifs (AP) et Affects Négatifs (AN), autorisant l'idée qu'il est possible de les ressentir en même temps (voir aussi Congard, Dauvier, Antoine, \& Gilles, 2011). Pour intégrer ces deux conceptions, c'est-à-dire considérer un espace où $\mathrm{AP}$ et $\mathrm{AN}$ sont indépendants et où la variable Activation (ACT) est également prise en compte, il faut utiliser un espace en trois dimensions : AN, AP et ACT (Stanley \& Meyer, 2009). C'est dans un espace affectif tridimensionnel que seront réalisées les analyses, car il permet d'identifier la capacité à ressentir simultanément des affects de valences différentes, ce qui semble être un facteur de protection (Congard et al., 2011), tout en prenant en compte le niveau d'activation.

\subsection{Développement de l'expertise en régulation affective}

Au-delà du constat d'une augmentation du bien-être affectif général (Cacioppo et al., 2008 ; Carstensen, Turan, Scheibe, Ram, Ersner-Hershfield, Samanez-Larkin, Brooks, \& Nesselroade, 2011 ; Gross et al., 1997 ; Guillaume et al., 2009), Mroczek et Kolarz (1998) ont montré que le ressenti des affects négatifs diminue jusqu'à l'âge de 60 ans environ, 
puis se stabilise, tandis que la fréquence des affects positifs augmente légèrement. Cette diminution des affects négatifs est également retrouvée par Charles, Reynolds et Gatz (2001). Ce phénomène est appelé « effet de positivité ». Il peut se traduire soit par une plus grande saillance des affects positifs, soit par une diminution des affects négatifs alors que celle des positifs reste stable avec l'âge (pour synthèse voir Vieillard \& Harm, 2013). Cet effet serait expliqué par une meilleure régulation affective, avec un changement d'objectif dans l'utilisation des stratégies de régulation qui seraient davantage axées sur les antécédents des affects chez les personnes âgées (Gross et al., 1997 ; Voelkle, Ebner, Lindenberger, \& Riediger, 2013).

\subsubsection{Sélectivité des stratégies}

Le modèle de sélectivité socioémotionnelle (SST) de Carstensen (1995) propose une explication de l'effet de positivité. Les personnes âgées seraient plus efficientes en régulation affective, car étant plus motivées elles engageraient plus de ressources dans cette activité et utiliseraient des stratégies plus efficaces (Carstensen et al., 1998). Elles développeraient une capacité à sélectionner les stratégies les plus efficaces par rapport à la situation et, surtout, choisiraient celles qui permettent de générer des affects positifs. Cette volonté de ressentir des affects positifs serait elle-même liée à la perception que le temps de vie futur est relativement court, incitant à une approche hédoniste. Ainsi, les individus âgés donneraient la priorité aux buts liés à l'expérience affective immédiate et à la régulation. Dans cette approche, ce n'est pas l'âge en soit qui expliquerait l'augmentation des affects positifs, mais l'impression subjective du temps restant devant soi. C'est-à-dire un état d'esprit particulier qui influence la perception affective et donne des objectifs différents, conduisant les personnes âgées à se centrer sur le ressenti d'affects positifs. Il faut noter que cette régulation n'est pas automatique et qu'elle nécessite des ressources cognitives telles que des ressources attentionnelles ou des fonctions exécutives comme l'inhibition ou la planification. Ainsi, lorsque les contraintes temporelles sont prégnantes, comme c'est le cas chez les plus âgés, il conviendrait d'éviter les expériences négatives devenues moins nécessaires et perçues davantage comme des sacrifices à court terme motivés par des objectifs de long terme (Fredrickson \& Carstensen, 1990). Les personnes âgées alloueraient davantage de ressources à l'utilisation des stratégies de régulation (Charles \& Pasupathi, 2003 ; Röcke, Li, \& Smith, 2009), car elles seraient plus motivées pour réduire le temps et l'énergie dépensés suite à des expériences négatives, particulièrement les événements sociaux déplaisants comme par exemple ceux liés à la construction d'un réseau social (Charles 
\& Carstensen, 2008). Au contraire, chez les personnes plus jeunes, lorsque le temps à venir est perçu comme sans limitation, les buts convergeraient vers l'accumulation d'informations et de connaissances nouvelles. Ces situations sont davantage susceptibles d'engendrer des expériences bénéfiques à long terme, mais potentiellement négatives à court terme telles que le passage d'examens qui peut engendrer du stress, de l'anxiété ou encore de l'irritation au moment des révisions, mais qui vise l'obtention d'un diplôme et d'un emploi dans le futur.

\subsubsection{Optimisation des ressources}

Un autre modèle est proposé pour expliquer la plus grande efficacité de la régulation affective chez les personnes âgées (Urry \& Gross, 2010). Il s'agit du modèle Sélection, optimisation et compensation en régulation émotionnelle (SOC-ER), qui est une adaptation directe du SOC Métathéorie développé par Baltes et Baltes en 1990. Le SOC-ER explique que les individus sélectionnent et optimisent certaines stratégies de régulation en tenant compte de leurs propres capacités (sélection), du contexte, de la pratique et des efforts à faire pour atteindre l'objectif fixé, ici l'augmentation ou la diminution de certains affects (optimisation). En fonction des résultats obtenus, ils développent des solutions de contournement ou de demande d'aide (compensation). Ainsi, Urry et Gross (2010) suggèrent que les personnes âgées compensent la diminution de leurs compétences (ressources cognitives) en favorisant et en optimisant certaines stratégies de régulation. Cette dernière idée complète la théorie de la Sélection socioémotionnelle (SST) vue précédemment.

\subsubsection{Prévention des affects négatifs par l'utilisation de stratégies proactives}

On peut imaginer que les personnes âgées, au-delà d'avoir développé la capacité à sélectionner la stratégie efficace (SST) et d'avoir optimisé leurs ressources pour la mettre en place (SOC-ER), soient aussi capables de prévenir la survenue d'un épisode affectif négatif. Gross et Thompson (2007) mettent en avant deux catégories de stratégies : les stratégies proactives, dont l'objectif est de modifier les informations responsables d'un affect avant qu'il émerge, et les stratégies réactives, qui consistent à modifier au moins l'une des trois composantes (expressive, cognitive ou physiologique) de la réponse émotionnelle après qu'elle ait été générée. Voelkle et ses collaborateurs (2013) distinguent la régulation proactive, dans laquelle la personne met en place des stratégies en amont de la survenue de l'affect dans le double but de limiter les affects négatifs et 
d'exacerber des affects positifs, et la régulation réactive, pour laquelle la mise en ouvre des stratégies suit la survenue de l'affect. On peut supposer que les personnes âgées acquièrent l'expérience suffisante leur permettant d'anticiper l'épisode affectif et, ainsi, d'utiliser plus de stratégies proactives. Etre proactif dans son comportement, et notamment dans la résolution de problème, amène à être plus efficace. Il s'agit de décider par anticipation des événements de vie souhaités. On peut parler d'un contrôle de l'environnement (par exemple avec le coping centré sur le problème) et de la manière dont ces événements sont vécus, ce qui renvoie à une forme de contrôle de soi-même (par exemple avec la réévaluation cognitive). Cette conception se traduit principalement par une prise d'initiative tendant à rompre avec les conduites habituelles de réponse à l'environnement, c'est-à-dire avec une régulation réactive (Bandura, 1999). Prenda et Lachman (2001) ont observé que l'anticipation du futur et sa préparation ont un effet bénéfique sur la satisfaction de vie, ce phénomène étant majoré chez leurs participants plus âgés.

\subsection{Objectifs et hypothèses}

L'objectif de cette recherche est d'étudier l'influence sur les affects du développement d'une forme d'expertise au niveau de la sélection et de l'utilisation de stratégies de régulation affective. Dans le prolongement des modèles de Sélectivité socioémotionnelle (Carstensen, 1995) et de Sélection, optimisation et compensation en régulation emotionnelle (Urry \& Gross, 2010), les expériences vécues conduiraient à la construction d'une base de connaissances sur l'efficacité des stratégies en fonction des situations qui s'étendrait avec l'âge. La personne apprendrait d'abord à utiliser différentes stratégies (ici les stratégies de coping centrées sur le problème, le soutien social, l'évitement, la réévaluation cognitive, la suppression expressive et la rumination) puis, avec la répétition, acquerrait des connaissances au niveau de leur efficacité à court et à long terme. Elle développerait progressivement une capacité à comprendre quand, où et comment chaque stratégie peut être appliquée et à différencier le caractère adaptatif de chacune. Ce processus serait lui-même renforcé par une allocation croissante de ressources au maintien des affects positifs conformément à la SST de Carstensen (1995). Si l'avancée en âge conduit au développement d'une expertise, alors en vieillissant l'individu développe sa capacité à sélectionner la stratégie de régulation adaptée à la situation, ainsi que ses capacités à superviser et à contrôler sa mise en œuvre. Les progrès dans l'utilisation même d'une stratégie donnée devraient se 
traduire par une baisse des affects négatifs et/ou une augmentation des affects positifs plus marquée(s) quand elle est mise en œuvre.

Ainsi, nous avons cherché à étudier le lien qui pourrait exister entre l'âge et les stratégies de régulation affective. Les adultes plus âgés privilégieraient certaines stratégies qu'ils percevraient comme plus efficaces (le coping centré sur le problème et la réévaluation cognitive). Ces mêmes stratégies évolueraient afin de devenir de plus en plus efficaces, les personnes intégrant de plus en plus d'informations à leur sujet grâce à leur utilisation répétée.

\section{MÉTHODE}

\subsection{Participants}

L'échantillon est constitué de 172 adultes tout-venants, âgés de 18 à 70 ans, dont 111 femmes. La moyenne d'âge est de 39 ans et l'écart-type de quinze ans et six mois. L'échantillon comprend 23 participants âgés de 18 à 21 ans, 45 de 22 à 31 ans, 31 de 32 à 41 ans, 25 de 42 à 51 ans, 30 de 52 à 61 ans et 18 de plus de 62 ans. L'âge chronologique a été retenu comme variable explicative reflétant à la fois l'expérience passée et le temps de vie future probable. En effet, l'âge apparaît comme étant un prédicteur fiable de la perception du temps futur telle que le conçoit le modèle de la sélection socioémotionnelle de Carstensen (1995), avec une forte corrélation négative $(r=-0,70 ; p<0,001$; Lang \& Carstensen, 2002). Recueillir l'âge au lieu de la perception du temps futur est aussi économique en temps de passation, car évaluable avec une seule question. Une estimation directe du temps de vie futur pourrait se différencier de l'âge chronologique pour des sujets âgés, mais une telle mesure constituerait une métrique difficilement interprétable sur un empan d'âge s'étendant aux jeunes adultes. Les participants ont été contactés par réseaux dans différentes régions de France (notamment Bretagne, Lorraine et Provence-AlpesCôte-d'Azur).

\subsection{Procédure et matériel}

La méthode de recueil de données adoptée permet de considérer des aspects temporels (les fluctuations des affects). Il s'agit d'un protocole de sondages en situation de vie quotidienne de type Experience Sampling Method (ESM), qui permet d'étudier les affects de manière relativement écologique et qui offre la possibilité d'étudier leurs fluctuations temporelles à court terme. Cette procédure est devenue la norme dans ce domaine (Wilt et al., 2011 ; Hektner, Schmidt, \& Csikszentmihalyi, 2006 ; Bolger \& Laurenceau, 2013). Les participants ont donné leur accord à cette étude en signant un formulaire de consentement, 
exposant les modalités de participation ainsi que la possibilité de se retirer à tout moment de l'étude. Les personnes n'ayant pas de forfait téléphonique adapté étaient dédommagées par un chèque cadeau d'une valeur de quinze euros. Le recueil de données est construit en deux étapes : l'entretien initial a pour but d'établir la liste des items qui serviront pour le recueil quotidien ; celui-ci s'effectue ensuite par l'intermédiaire de SMS grâce au téléphone portable des participants.

\subsubsection{La définition des items}

Un entretien individuel était réalisé afin de construire la liste d'items utilisée lors de la phase de sondage quotidien. La procédure était identique pour les affects et les stratégies. Suite à la lecture de définitions ( $c f$. annexe 1), le participant choisit un adjectif par affect et une petite phrase ou un verbe par stratégie, qui décrit de manière la plus compréhensible pour lui les douze affects et les six stratégies de régulation. Les définitions des affects ont été construites à partir des douze cadrans du modèle en circomplexe de Yik et al. (2011) et celles des stratégies à partir des éléments théoriques évoqués en introduction. Cette procédure permet de réaliser, pour chaque participant, une liste de douze adjectifs correspondant aux états affectifs et de six petites phrases désignant les stratégies de régulation : ces 18 éléments constituent les items auxquels la personne répond dans la phase de sondages quotidiens. Afin de s'assurer de la concordance entre les items choisis par les participants et les affects et stratégies ciblés, une procédure de vérification a été mise en place : à la fin de l'entretien, chaque item choisi était présenté par l'expérimentateur, le participant devant alors restituer la signification qu'il lui associait. Ce procédé permet de limiter le biais que peut représenter des significations différentes d'un même terme pour deux participants différents (Nesselroade, Gerstorf, Hardy, \& Ram, 2007).

\subsubsection{Le recueil quotidien par SMS}

L'entretien individuel initial a aussi permis de définir la période de recueil de données. Il s'agissait de déterminer une durée de quatorze jours consécutifs où les participants répondraient aux 18 items sélectionnés (douze affects et six stratégies), toutes les trois heures, cinq fois par jour, en réponse à un SMS de rappel. Chaque participant pouvait ajuster l'horaire auquel le message d'alerte était envoyé, dans un intervalle de plus ou moins quinze minutes autour des cinq moments quotidiens (généralement $9 \mathrm{~h}, 12 \mathrm{~h}, 15 \mathrm{~h}, 18 \mathrm{~h}$ et $21 \mathrm{~h}$ ). Un délai de 30 minutes pour répondre était accordé après réception du message de rappel. Ces messages étaient envoyés de manière automatique à partir d'un serveur. Ainsi, cinq fois par jour, les participants recevaient sur leur téléphone portable le message suivant : «Étude sur les émotions, veuillez répondre dans un délai de 30 minutes en suivant les consignes de votre fiche. Merci à vous. ». Ils utilisaient alors la fonction « répondre » de leur téléphone portable. Pour les items concernant les affects, il leur était demandé d'indiquer leur niveau de ressenti au moment de la réponse, tandis que pour les stratégies, la consigne portait sur le niveau d'utilisation estimé de chaque stratégie durant la 
période temporelle écoulée depuis la réponse précédente. Les réponses se faisaient sur une échelle d'évaluation allant de 1 « pas du tout » à 5 «beaucoup » (c'est-à-dire ne pas ressentir du tout tel affect $v s$. le ressentir beaucoup en ce moment ; ne pas avoir du tout utilisé la stratégie de régulation depuis la dernière réponse vs. l'avoir utilisé beaucoup). Il n'était pas demandé aux participants de faire un lien entre les affects ressentis et les stratégies et ils étaient d'autant moins incités à le faire que les questions sur les affects étaient antérieures à celles sur les stratégies. Pour répondre, les participants envoyaient un SMS comprenant uniquement les chiffres de la modalité de l'échelle de réponse choisie pour chaque item (soit 18 chiffres pour les 18 items, regroupés en six paquets de trois pour éviter au maximum les erreurs de frappe).

Une fois l'ensemble des données obtenues, nous avons enlevé les réponses comportant une erreur de frappe (par exemple insertion d'un $19^{\mathrm{e}}$ chiffre dans la séquence), les doublons, les réponses ayant un ou plusieurs chiffre(s) manquant(s), ainsi que les réponses arrivant avec un intervalle de temps inférieur à $2 \mathrm{~h} 15$ par rapport aux SMS précédents et suivants. Les réponses des dix participants ayant répondu à moins de la moitié des 70 observations prévues ont également été retirées. De plus, nous avons ôté les réponses de cinq participants ayant un niveau d'affectivité négative particulièrement élevé sur l'ensemble de la période d'observation. Sur les 70 SMS envoyés à chaque participant pendant la durée de l'étude, nous avons reçu en moyenne 60 réponses par participant, soit un taux de $86 \%$.

\section{RÉSULTATS}

\subsection{Structure factorielle de l'espace des affects}

Les réponses aux douze items d'affects pour chaque SMS ont été soumises à une analyse en composantes principales dans le but de vérifier si l'on pouvait retrouver les trois grands axes : Affectivité Positive, Affectivité Négative et Activation. L'objectif de cette analyse est de projeter les réponses aux douze affects dans un sous-espace de dimensions plus réduites. Les facteurs extraits ne sont donc pas interprétés comme des variables latentes basées sur des processus psychologiques distincts. Il s'agit uniquement d'une étape de réduction dimensionnelle permettant de dégager des variables dépendantes sous la forme de trois scores factoriels. À ce niveau, les variabilités intra et interindividuelles ne sont pas distinguées. L'analyse du graphique des éboulis aboutit effectivement à retenir trois composantes dont les valeurs propres sont supérieures à un et qui résument $64 \%$ de la variance totale. Les cartes factorielles projetant les items dans l'espace des composantes obtenues montrent bien une organisation en arc de cercle compatible avec le modèle circomplexe. Ce patron apparait également 
en considérant la troisième composante. Cette organisation circulaire des items fait que les rotations habituelles comme la rotation Varimax sont difficiles à utiliser. Le tableau 1 montre les saturations obtenues à la suite d'une rotation ad hoc ${ }^{1}$ réalisée dans le but de dégager trois scores factoriels identifiables comme essentiellement influencés par les affects positifs, les affects négatifs et le niveau d'activation.

Le premier facteur rassemble l'inactivation déplaisante, le déplaisir inactif, le déplaisir, le déplaisir actif et l'activation déplaisante. Nous avons choisi d'intituler cette dimension Affectivité Négative ou AN. Le plaisir, le plaisir inactif et l'inactivation plaisante constituent le deuxième facteur. Le dernier facteur présente des saturations positives avec l'activation, l'activation plaisante, le plaisir actif et une négative avec l'inactivation. Les facteurs deux et trois sont appelés par la suite respectivement Affectivité Positive (AP) et Activation (ACT). La composante AP est légèrement déséquilibrée vers le pôle basse-activation. Ainsi les AP font un peu plus référence à de la sérénité qu'à un état positif neutre d'activation, tel que le modèle circomplexe de Russell l'a définie. L'activation comporte quant à elle un aspect de valence positive. La distribution des AN est asymétrique (voir le coefficient d'asymétrie Tableau 1), les valeurs élevées sont dans l'ensemble peu fréquentes : nous l'avons normalisée en prenant le logarithme népérien des scores factoriels.

Les corrélations calculées entre les scores factoriels à partir des moyennes individuelles (tableau 3 montrent un lien négatif modéré entre AP et AN $(r=-0,35 ; p<0,01)$ ce qui est conforme aux résultats de la littérature. Au niveau des moyennes individuelles, l'activation apparait quant à elle, liée avec les AP $(r=0,58 ; p<0,01)$, mais pas avec les AN $(r=-0,14 ; \mathrm{ns})$.

\subsection{Liens entre l'âge, les affects et l'utilisation des stratégies}

Les corrélations entre l'âge et les affects semblent indiquer un effet de positivité d'ampleur modéré (Tableau 3). L'âge est corrélé positivement avec le niveau d'AP $(r=0,18 ; p<0,05)$ et le niveau d'activation qui comporte des items d'Affectivité positive $(r=0,24 ; p<0,01)$. L'âge est aussi

\footnotetext{
${ }^{1}$ Le nuage de points des scores factoriels sur les deux premières composantes des ACP réalisées sur des mesures d'affects présente généralement une forme proche d'un triangle rectangle. Le sommet en angle droit du triangle correspond aux réponses avec des niveaux faibles sur tous les adjectifs (ni joyeux, ni triste) et les deux autres sommets correspondent aux valeurs élevées sur les adjectifs d'AP et d'AN respectivement. La rotation opère de manière à aligner les deux premières composantes sur les côtés de ce triangle.
} 
Tableau 1. Saturations de l'analyse en composantes principales des douze états affectifs après rotation, données descriptives des affects et corrélations avec l'âge

Table 1. Loadings of principal components analysis on the affects (SMS) after rotation, descriptive data of affects and correlations with age

\begin{tabular}{|c|c|c|c|c|c|}
\hline Affect & AN & AP & ACT & $\begin{array}{l}\text { Moyenne } \\
\text { (ET) }\end{array}$ & $\begin{array}{l}\text { Corrélations } \\
\text { avec l'âge }\end{array}$ \\
\hline Activation plaisante & $-0,37$ & 0,38 & 0,78 & $3,01(1,14)$ & $0,32^{* *}$ \\
\hline Plaisir actif & $-0,42$ & 0,58 & 0,70 & $2,96(1,15)$ & 0,14 \\
\hline Plaisir & $-0,49$ & 0,71 & 0,55 & $3,24(1,09)$ & 0,07 \\
\hline Plaisir inactif & $-0,36$ & 0,83 & 0,26 & $3,13(1,16)$ & $0,30^{* *}$ \\
\hline Inactivation plaisante & $-0,36$ & 0,84 & 0,16 & $3,15(1,20)$ & 0,13 \\
\hline Inactivation & 0,44 & 0,16 & $-0,44$ & $2,10(1,22)$ & $-0,21^{* *}$ \\
\hline Inactivation déplaisante & 0,73 & $-0,29$ & $-0,32$ & $1,61(0,98)$ & $0,14^{*}$ \\
\hline Déplaisir inactif & 0,80 & $-0,46$ & $-0,07$ & $1,44(0,82)$ & $-0,11$ \\
\hline Déplaisir & 0,77 & $-0,46$ & $-0,03$ & $1,60(0,95)$ & $-0,13^{*}$ \\
\hline Déplaisir actif & 0,74 & $-0,54$ & 0,06 & $1,63(0,98)$ & $-0,12$ \\
\hline Activation déplaisante & 0,71 & $-0,52$ & 0,10 & $1,68(1,00)$ & $-0,15^{\star}$ \\
\hline Activation & 0,02 & 0,08 & 0,72 & $2,07(1,20)$ & 0,13 \\
\hline Coefficient asymétrie & 1,26 & 0,04 & $-0,26$ & & \\
\hline
\end{tabular}

lié négativement à la dimension d'Affectivité négative $(r=-0,20 ; p<0,01)$. Les corrélations reportées dans le tableau 1 entre l'âge et chacun des affects vont dans le même sens, avec des corrélations entre l'âge et le Plaisir inactif $(r=0,30 ; p<0,01)$ et le Plaisir inactif $(r=0,32 ; p<0,01)$ ainsi qu'entre l'âge et l'Activation déplaisante $(r=-0,15 ; p<0,05)$ et l'inactivation $(r=-0,21 ; p<0,05)$. Avec l'avancement en âge, les individus semblent privilégier certaines stratégies. Deux stratégies paraissent plus fortement utilisées avec l'âge : le coping centré sur le problème $(r=0,22 ; p<0,01)$ et la réévaluation cognitive $(r=0,21 ; p<0,01)$. Nous avons vu que ces stratégies sont généralement reconnues dans la littérature comme étant efficaces. De plus, le lien avec les AP est positif pour la réévaluation cognitive $(r=0,48$; $p<0,01)$ et le coping centré sur le problème $(r=0,41 ; p<0,01)$. Enfin, le niveau d'activation est nettement lié à la réévaluation cognitive $(r=0,41$; $p<0,01)$ et au coping centré sur le problème $(r=0,51 ; p<0,01)$.

Il apparaît aussi que l'utilisation de quatre des six stratégies de régulation, entre autres le coping centré sur le problème qui est une stratégie efficace, est liée positivement avec les affects négatifs, ce qui semble 


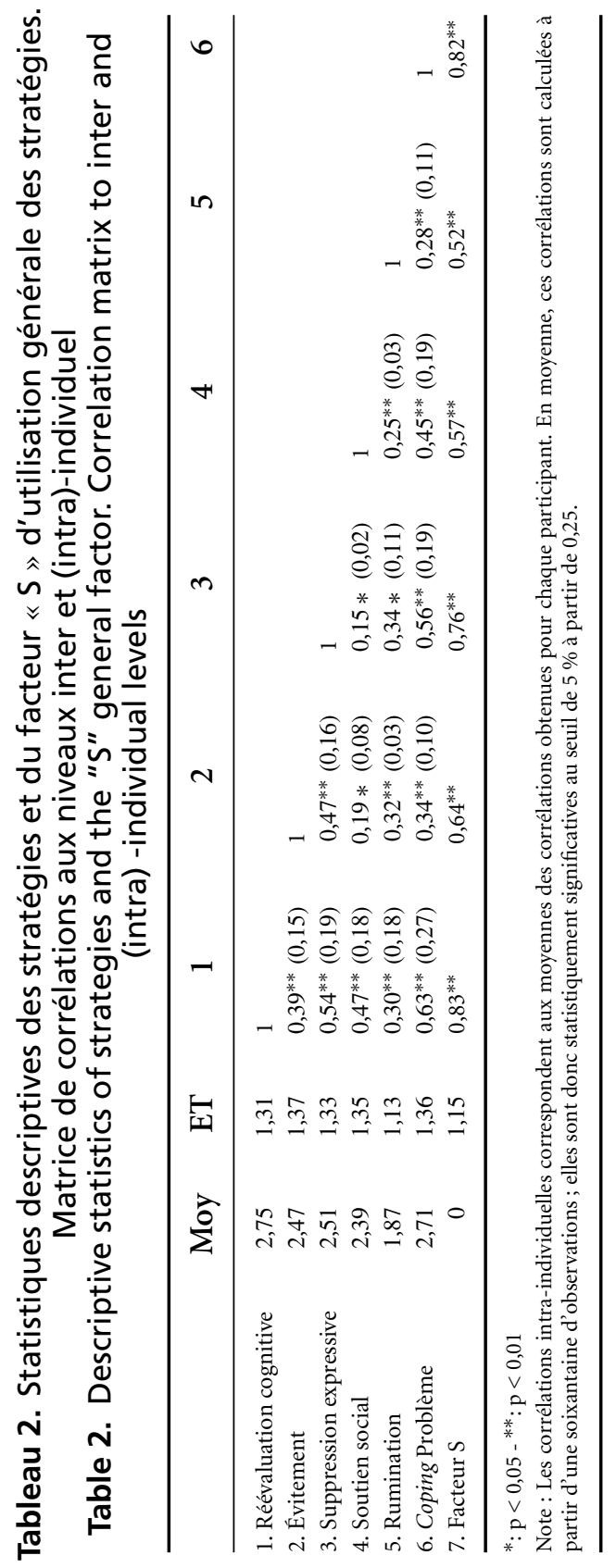


contre-intuitif puisque les AP et les AN sont corrélés négativement $(r=$ $-0,35 ; p<0,01)$. Ce phénomène pourrait refléter l'impact des événements de vie rencontrés. En effet sur la période de quatorze jours de recueil, il est possible que les participants aient rencontré plus ou moins de situations de vie nécessitant la mise en œuvre de stratégies de régulation. Une personne confrontée à des événements négatifs devrait ressentir davantage d'affects négatifs, ce qui la conduirait à utiliser davantage de stratégies de régulation et expliquerait les corrélations positives observées entre affectivité négative et stratégies dites adaptées. Il est probable que le facteur $S$, qui est le facteur général d'utilisation des six stratégies, soit en partie influencé par le contenu des expériences de vie. Cette interprétation est confortée par la corrélation partielle obtenue entre l'âge et les AN en contrôlant l'effet du facteur S ( $r$ part. $=-0,27 ; p<0,01)$ qui apparaît plus marquée que la corrélation simple $(r=-0,20 ; p<0,01)$. Tout se passe comme si, à situation de vie comparable, les personnes plus âgées ressentaient moins d'affects négatifs. Dans le même ordre d'idée, la corrélation négative entre l'âge et la rumination, souvent identifiée comme moins efficace dans la littérature, devient significative si l'on contrôle le facteur $S$ d'utilisation générale des stratégies ( $r$ part. $=-0,26 ; p<0,01)$, conformément à l'hypothèse de l'expertise.

\subsection{Les stratégies de régulation}

Les six stratégies de régulation (coping centré sur le problème, soutien social, évitement, réévaluation cognitive, suppression expressive et rumination) ont été résumées par leur niveau moyen d'utilisation par sujet sur l'ensemble de la période d'observation pour calculer les statistiques descriptives présentées dans le Tableau 2. D'après les moyennes globales, on observe des niveaux d'utilisation des stratégies assez homogènes, sauf pour la rumination qui apparaît sensiblement moins utilisée. Nous pouvons supposer que, puisqu'elle n'est pas jugée à long terme comme efficace (Luminet, 2002), les participants y ont moins recours. Ce sont la réévaluation cognitive et le coping centré sur le problème qui apparaissent comme étant les plus fortement utilisés au niveau interindividuel. Ce sont aussi ces stratégies qui sont désignées comme les plus efficaces dans la littérature. Sur le plan des corrélations obtenues au niveau des moyennes par sujet, l'utilisation d'une stratégie est très souvent liée à l'utilisation des autres, notamment la réévaluation cognitive avec le coping centré sur le problème $(r=0,63 ; p<0,01)$. Les corrélations obtenues au niveau intra-individuel, c'est-à-dire la moyenne des corrélations calculées séparément pour chaque sujet entre chacune des stratégies, sont sensiblement plus faibles qu'au niveau interindividuel (Tableau 2) ce qui 
va dans le sens d'une distinction entre les stratégies. Certains participants ont utilisé plus que d'autres l'ensemble des six stratégies sans pour autant les utiliser toutes au même moment. Les corrélations entre les stratégies laissent supposer l'existence d'un facteur général, renvoyant à l'utilisation de stratégies de façon plus globale, désigné sous le terme de «facteur $\mathrm{S}$ », identifié ici à l'aide d'une analyse en composantes principales réalisée sur l'ensemble des observations. Elle a permis de dégager un facteur stratégique général qui apparaît majoritairement lié, au niveau des moyennes par sujet, à l'utilisation de la réévaluation cognitive $(r=0,83 ; \mathrm{p}<0,01)$ et du coping centré sur le problème $(r=0,82 ; \mathrm{p}<0,01)$, mais aussi de manière substantielle aux autres stratégies y compris la rumination $(r=0,57$; $\mathrm{p}<0,01)$. Ce facteur refléterait ainsi la possibilité pour les participants d'utiliser plusieurs stratégies dans un même intervalle temporel.

\section{4. Évolution de l'efficacité des stratégies avec l'âge}

Notre deuxième hypothèse vient interroger le développement d'une expertise dans l'utilisation des stratégies privilégiées qui influencerait plus fortement les affects. Cette expertise se traduit par l'interaction de l'âge avec les stratégies utilisées entre $t$ et $t_{+1}$ sur les affects exprimés à $t_{+1}$. L'efficacité d'une stratégie est évaluée par l'augmentation ou la diminution des affects ressentis en fonction du niveau d'utilisation de cette stratégie, relation qui elle-même peut varier en fonction de l'âge. Les résultats obtenus au niveau interindividuel (Tableau 3) suggèrent que l'utilisation du coping centré sur le Problème (Prob) et de la Réévaluation cognitive (RC) est associée à un niveau élevé d'affects positifs. Nous avons vu précédemment qu'avec l'âge, les individus privilégieraient ces deux stratégies spécifiques. Si au cours du développement adulte, les personnes apprennent à mieux les utiliser, elles devraient les employer avec plus d'efficacité. Afin d'évaluer cet effet modérateur de l'âge dans la relation entre stratégies de régulation et affects, des modèles linéaires généralisés à effets mixtes $(\mathrm{GLMM})^{2}$ ont été estimés avec les trois dimensions affectives en variables à expliquer et les deux stratégies de régulation au niveau des observations (SMS) en interaction avec l'âge au niveau des sujets en variables explicatives. Pour éviter des effets de colinéarités entre les variables et leur produit représentant l'interaction et permettre d'obtenir des coefficients standardisés, les analyses ont été réalisées à partir des données centrées-réduites. De plus, nous avons

${ }^{2}$ Les Modèles linéaires généralisés à effets mixtes (GLMM) sont estimés à partir de la librairie lme4 du programme R (Bates, Maechler, Bolker, \& Walker, 2013). 
Tableau 3. Matrice de corrélations entre l'âge, les affects (AP, AN, $A C T)$, les moyennes individuelles des stratégies et le facteur $S$

Table 3. Correlation matrix between age, affects (PA, NA, ACT), individual strategy use means and $\mathrm{S}$ factor

\begin{tabular}{lcccc}
\hline & Age & AP & AN & ACT \\
\hline AP & $0,18^{*}$ & 1 & & \\
AN & $-0,20^{* *}$ & $-0,35^{* *}$ & 1 & \\
Act & $0,24^{* *}$ & $0,58^{* *}$ & $-0,14$ & 1 \\
Réévaluation cognitive & $0,21^{*}$ & $0,48^{* *}$ & 0,13 & $0,41^{* *}$ \\
Évitement & 0,04 & $0,18^{*}$ & $0,34^{* *}$ & $0,19^{*}$ \\
Suppression & 0,14 & $0,30^{* *}$ & $0,25^{* *}$ & $0,33^{* *}$ \\
Soutien social & 0,04 & $0,23^{* *}$ & 0,12 & $0,33^{* *}$ \\
Rumination & $-0,15$ & $-0,05$ & $0,64^{* *}$ & 0,11 \\
Coping problème & $0,22^{* *}$ & $0,41^{* *}$ & $0,15^{*}$ & $0,51^{* *}$ \\
Facteur S & $0,16^{*}$ & $0,41^{* *}$ & $0,47^{* *}$ & $0,34^{* *}$ \\
\hline
\end{tabular}

*:p $<0,05-* *: p<0,01$

systématiquement testé l'effet non linéaire de l'âge tel que le suggèrent Mroczek et Korlarz (1998), ainsi que l'effet non-linéaire de l'utilisation de la stratégie en introduisant les polynômes d'ordre deux dans les modèles $^{3}$. Au niveau intra-individuel, il est envisageable que la relation entre utilisation des stratégies et affects ressentis soit plus complexe qu'au niveau interindividuel. Pour une personne donnée, on s'attend à ce que l'utilisation de la réévaluation cognitive soit associée, par exemple, à un plus haut niveau d'affectivité positive, mais un faible niveau d'utilisation de cette stratégie ne serait pas nécessairement associé à un faible niveau d'AP. En effet, si l'environnement est clément, il est possible que les affects positifs soient élevés sans nécessité de mettre en ouvre la réévaluation. Les AP pourraient ainsi être élevés aux moments où les participants n'utilisent pas la réévaluation (car ce n'est pas nécessaire) et quand ils l'utilisent beaucoup (car c'est une stratégie efficace), ce qui conduit à un lien non-linéaire en U.

Nous avons donc testé six séries de modèles (trois affects * deux stratégies), en faisant varier la structure explicative depuis un modèle avec uniquement un intercept par participant, jusqu'à un modèle avec une interaction non-linéaire entre l'âge et l'utilisation de la stratégie (Tableau 4). À chaque fois, les meilleurs modèles obtenus sont ceux ayant l'âge et la stratégie en interaction non-linéaire (polynôme d'ordre 2) d'après le critère

\footnotetext{
${ }^{3}$ La fonction poly() de R permet d'obtenir la variable et sa transformation avec une corrélation nulle.
} 


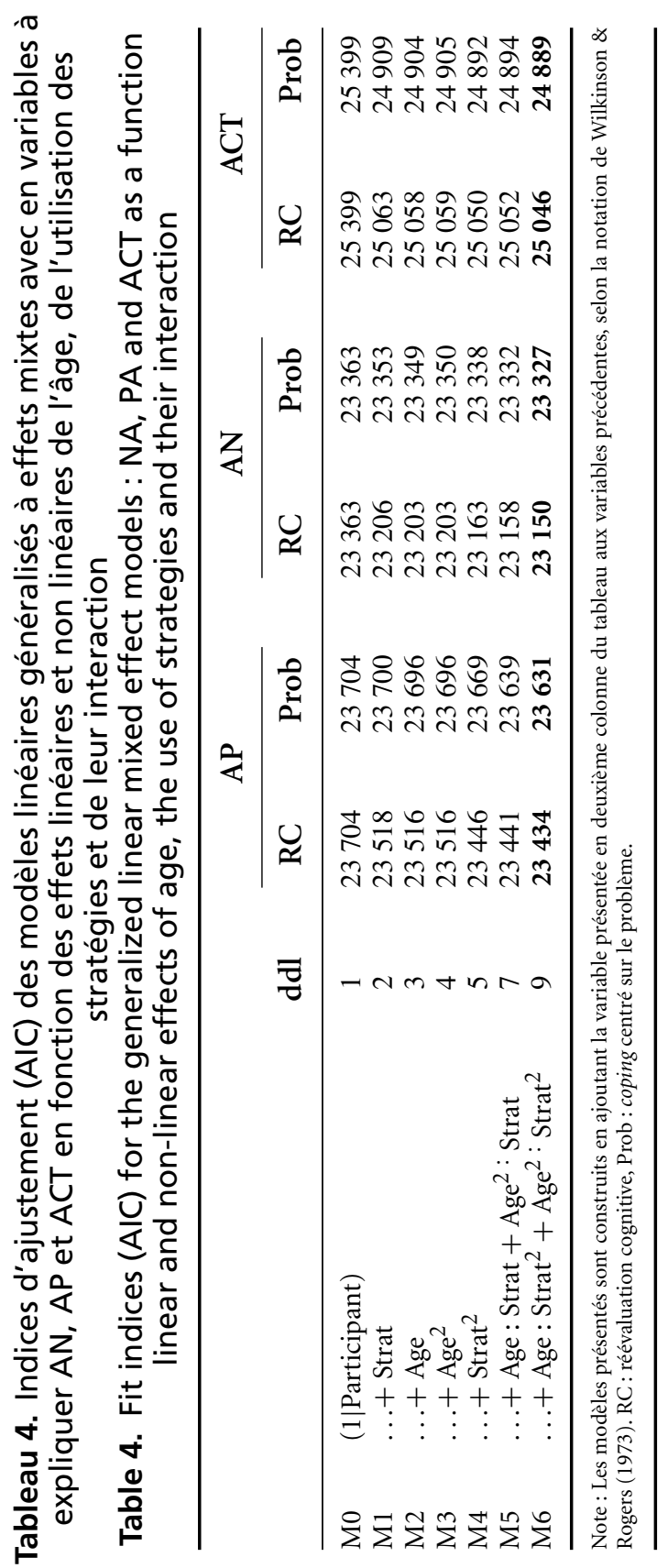


Tableau 5. Paramètres des 6 modèles estimés par GEE et $R^{2}$ des scores factoriels d'affects en fonction des effets linéaires et non linéaires de l'âge, de l'utilisation des stratégies RC et Prob, et de leur interaction

Table 5. Parameters of the 6 models fitted with GEE and $R^{2}$ as a function linear and non-linear effects of age, the use of strategies and their interaction

\begin{tabular}{|c|c|c|c|c|c|c|c|c|c|c|}
\hline & & AP & & & A & $\mathrm{N}$ & & & ACT & \\
\hline & $\mathrm{RC}$ & Prob & & $\mathrm{RC}$ & & Prob & & $\mathrm{RC}$ & Prob & \\
\hline Intercept & $-0,22$ & $-0,19$ & & 0 & $* *$ & 0,04 & * & 0,01 & 0,01 & \\
\hline & $-0,07$ & $-0,08$ & & $-0,09$ & & $-0,13$ & & 0,03 & 0,01 & \\
\hline Age $^{2}$ & 0,08 & 0,08 & & 0,08 & & 0,04 & & $-0,07$ & $-0,08$ & \\
\hline Strat & 0,20 & 0,02 & $* *$ & $-0,03$ & $* *$ & 0,16 & & 0,23 & 0,3 & $* *$ \\
\hline Strat $^{2}$ & 0,17 & 0,1 & $* *$ & $-0,15$ & $* *$ & $-0,19$ & & 0,04 & 0,01 & \\
\hline Age: Strat & $-0,02$ & $-0,04$ & & $-0,05$ & & $-0,02$ & & $-0,07$ & $-0,07$ & \\
\hline Age $^{2}:$ Strat & 0,04 & 0,1 & & 0,06 & & $-0,08$ & * & 0 & 0 & \\
\hline Age : Strat ${ }^{2}$ & 0,12 & 0,13 & & $-0,06$ & $* *$ & $-0,01$ & ** & 0,1 & 0,1 & $* *$ \\
\hline Age $^{2}:$ Strat $^{2}$ & $-0,02$ & 0,02 & & 0 & & 0,05 & & 0,03 & 0,06 & \\
\hline $\mathrm{R}^{2}$ & 0,13 & 0,08 & & 0,05 & & 0,05 & & 0,1 & 0,14 & \\
\hline
\end{tabular}

de l'AIC. Afin de se représenter le sens de cette évolution, nous avons réalisé des graphiques à partir de données prédites par des modèles estimés par la méthode d'estimation d'équations généralisées $(\mathrm{GEE})^{4}$, car les prédictions basées sur les effets fixes sont plus fiables avec cette procédure qu'avec les GLMM (Gardiner, Luo, \& Roman, 2009). Pour chacune des prédictions, nous avons calculé les niveaux d'affectivité positive, d'affectivité négative et d'activation en considérant des individus avec des âges de 24, 39, 54 et 69 ans (c'est-à-dire qui se situent respectivement à -1, 0,1 et 2 écarts-types de l'âge moyen), et différents niveaux d'utilisation des deux stratégies identifiées précédemment dans un intervalle de $\pm 1,6$ écart-type. Choisir un tel intervalle permet de considérer $90 \%$ de la distribution, qui est approximativement gaussienne. Les paramètres des modèles estimés par GEE utilisés pour les prédictions ainsi que les $\mathrm{R}^{2}$ associés sont présentés dans le Tableau 5. Les $\mathrm{R}^{2}$ peuvent paraître modestes, mais une valeur de $\mathrm{R}^{2}$ de 0,10 correspond à une corrélation de 0,33 , ce qui est l'ordre de grandeur

${ }^{4}$ Les Équations d'estimation généralisées (GEE) sont réalisées à partir de la librairie geepack du programme $\mathrm{R}$ (Hojsgaard, Halekoh, \& Yan, 2006 ; Yan \& Fine, 2004 ; Yan, 2002). 
de certaines corrélations entre âge et affects. De plus, ces modèles portent sur la variabilité intra-individuelle des affects qui ne peut s'expliquer que partiellement par l'utilisation d'une seule stratégie de régulation. Les représentations graphiques des prédictions (Figure 1) permettent de mieux se rendre compte de la forme des effets non linéaires et de l'interaction.

L'analyse des courbes obtenues (Figure 1) met en évidence trois éléments importants. Le premier concerne l'efficacité des stratégies lorsqu'elles sont utilisées de manière intense pour réduire les affects négatifs (parties droites des différents panneaux). Sur la droite des panneaux a et $b$, les courbes des plus jeunes atteignent le point le plus élevé lorsque les stratégies sont fortement utilisées. Le niveau d'AN atteint est donc le plus haut pour les participants plus jeunes. Lorsque les personnes plus âgées utilisent beaucoup la réévaluation ou le coping problème, leur niveau d'AN est plus bas alors que pour les plus jeunes, en revanche, les AN restent élevés même quand ils utilisent fortement ces deux stratégies. Symétriquement, l'analyse de la partie droite des panneaux relatifs aux affects positifs et à l'activation montre des niveaux plus élevés pour les plus âgés en cas d'utilisation intensive des deux stratégies. Ce patron de résultats, particulièrement net pour le coping centré sur le problème, va bien dans le sens d'une augmentation avec l'âge de l'efficacité des stratégies théoriquement les plus adaptées pour réduire les AN et augmenter les AP.

Le deuxième résultat important est en lien avec la forme curvilinéaire observée, notamment pour les affects positifs et l'activation. Cette forme pourrait s'expliquer par la causalité réciproque qui relie stratégies et affects. Si l'objectif d'une stratégie est de modifier le niveau d'affect, sa mise en œuvre n'est utile que si le niveau d'affect initial le nécessite. Autrement dit, si utiliser des stratégies permet d'aller mieux, il n'est pas nécessaire de les mettre en ouvre quand cela va déjà bien. Les niveaux d'AP peuvent donc être élevés à deux moments : quand les stratégies sont peu utilisées, car les affects positifs sont déjà élevés et quand l'utilisation d'une stratégie s'est avérée efficace pour les faire monter. Sur les panneaux $c$ et $\mathrm{d}$ de la figure 1 pour l'affectivité positive, mais aussi sur les panneaux e et $\mathrm{f}$ pour l'activation, on constate que cette forme en $\mathrm{U}$ est plus marquée pour les plus âgés, alors que les courbes sont quasiment linéaires chez les plus jeunes. Les quatre courbes s'en trouvent différenciées en fonction de l'âge sur leur extrémité gauche. En particulier, on observe que seuls les plus jeunes expérimentent des moments d'affectivité positive et d'activation faibles, sans pour autant mettre en œuvre de façon plus intense les stratégies de régulation. Tout se passe comme si un faible niveau d'affectivité positive ou d'activation conduisait les personnes expérimentées à utiliser des stratégies de régulation, alors que les plus jeunes ne percevaient pas cet indice. 

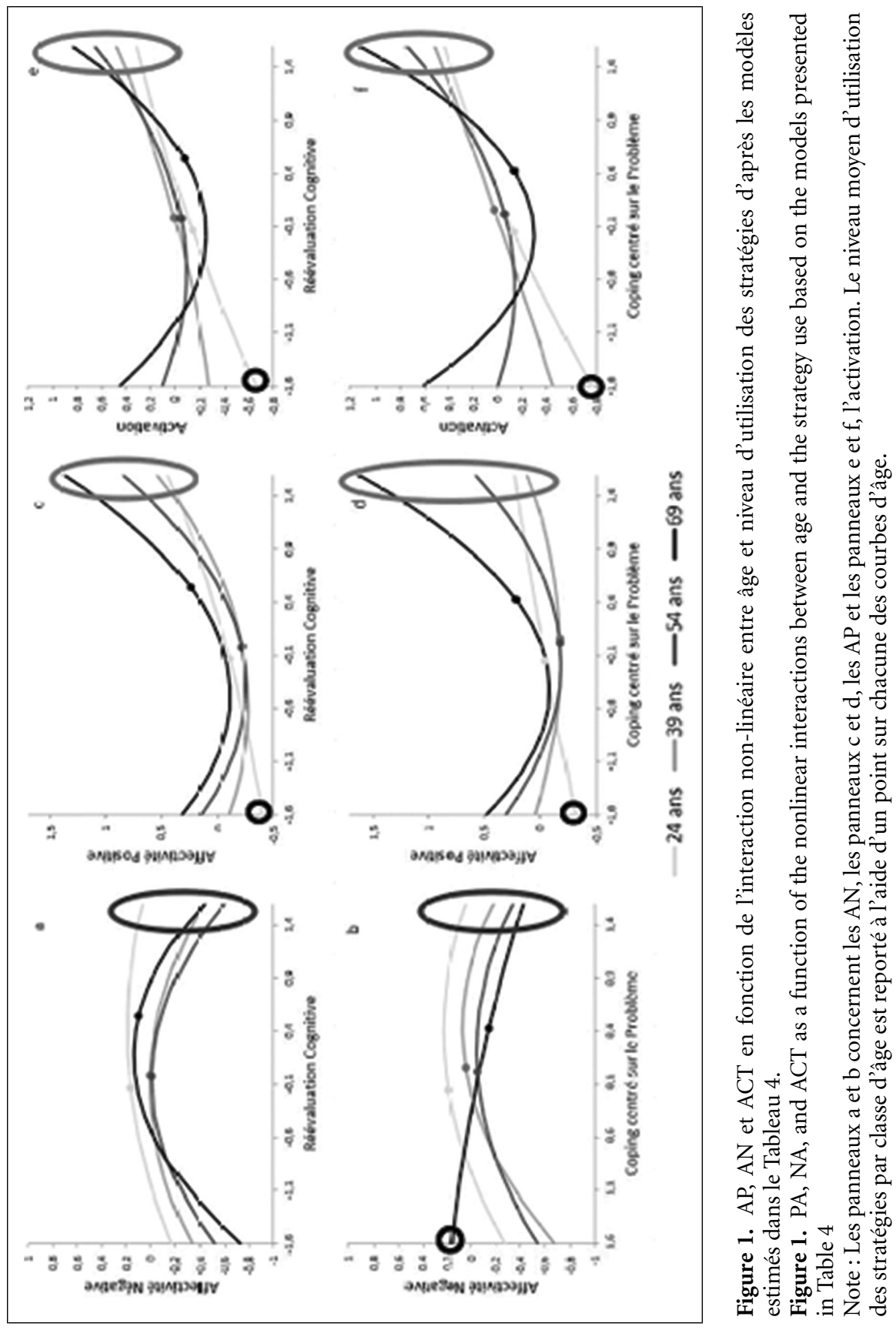
Le troisième résultat remarquable concerne spécifiquement l'effet du coping centré sur le problème sur les affects négatifs (panneaux b, Figure 1). On constate que, pour les plus âgés, le niveau d'affectivité négative est particulièrement élevé dans les moments où le coping centré sur le problème n'est pas mis en œuvre. Tout se passe comme si le fait de ne pas mobiliser cette stratégie les rendait plus vulnérables. Cela ne semble pas se produire pour les adultes plus jeunes, entre 30 et 50 ans. Quand ces derniers n'utilisent pas le coping centré sur le problème, le niveau d'AN est faible, comme s'ils mettaient en œuvre cette stratégie en réaction à des événements de vie générant des AN. Le coping centré sur le problème semble donc avoir un statut différent en fonction de l'âge : il serait utilisé chez les adultes d'âge intermédiaire de manière plus réactive en réponse à des événements de vie, alors que chez les adultes plus âgés, il deviendrait une condition nécessaire pour contenir le niveau d'affectivité négative. Le fait que le niveau d'utilisation du coping centré sur le problème augmente avec l'âge est aussi en cohérence avec cette interprétation.

\section{DISCUSSION}

L'objectif de cette étude était d'étudier l'influence sur les affects du développement d'une forme d'expertise au niveau de la sélection et de l'utilisation de stratégies de régulation au cours de l'âge adulte, à l'aide d'une méthodologie originale sur deux aspects : un recueil de données par sondages quotidiens en situation de vie quotidienne qui permet de mieux appréhender les fluctuations inter et intra-individuelles d'un questionnaire en passation transversale et l'approche vie entière qui permet d'analyser l'effet de l'âge comme une variable continue plutôt qu'en procédant par comparaison de groupes. Plus précisément, cette étude a permis d'analyser la variabilité interindividuelle et développementale chez des participants de 18 à 70 ans et la variabilité intra-individuelle avec des mesures répétées sur une période de deux semaines.

Parmi les nombreux aspects des processus de régulation affective, de leur évolution et de leur efficacité en fonction de stratégies évoquées dans la littérature, nous avons choisi de nous focaliser sur la sélection et l'efficacité de certaines stratégies en fonction de l'âge. Les résultats font apparaître un effet de positivité d'amplitude modérée. Conformément aux résultats antérieurs, les affects positifs ressentis augmentent légèrement avec l'âge dans un même ordre de grandeur que celui décrit dans la 
littérature avec une méthodologie semblable (Scheive, English, Tsai, \& Carstensen, 2013 ; Carstensen, Pasupathi, Mayr, \& Nesselroade, 2000 ; Stone, Schwartz, Broderick, \& Deaton, 2010). L'avancée en âge semble également s'accompagner d'une baisse des affects négatifs. Ces résultats pourraient s'expliquer par une meilleure sélection des stratégies de régulation émotionnelle, comme le proposent les modèles de la Sélection socioémotionnelle (Carstensen et al., 1998) et de la Sélection, optimisation et compensation en régulation émotionnelle (Urry \& Gross, 2010). Parmi les six stratégies investiguées dans cette étude (coping centré sur le problème, évitement, soutien social, réévaluation cognitive, suppression expressive et rumination), nous avons pu observer qu'avec l'âge les personnes privilégieraient deux stratégies de régulation affective : le coping centré sur le problème et la réévaluation cognitive. Ces deux stratégies apparaissent également liées à un niveau d'activation important ce qui peut faire supposer qu'elles demandent un certain niveau d'engagement et de dynamisme de la part des participants pour être utilisées. Différentes études en laboratoire avaient aussi montré que les personnes âgées utilisent plus la réévaluation positive que les plus jeunes (par ex. Shiota \& Levenson, 2009). Ainsi, avec l'avancée en âge les personnes auraient tendance à utiliser plus fortement certaines stratégies de régulation qu'ils percevraient comme plus efficaces, illustrant un phénomène de sélection au sein du répertoire stratégique.

En plus des liens entre l'âge et la sélection des stratégies, nous avons mis en évidence un effet développemental sur l'efficacité même des stratégies privilégiées. Ceci est attendu dans le cadre du modèle de la Sélection socioémotionnelle (Carstensen, et al., 1998), car selon ce modèle, les personnes plus âgées alloueraient davantage de ressources personnelles, notamment motivationnelles, à la régulation affective. La modélisation de l'interaction non-linéaire entre l'âge et les stratégies sur les trois dimensions affectives (affectivité positive, affectivité négative et activation) met en évidence trois éléments. Premièrement, les deux stratégies privilégiées avec l'âge permettraient effectivement aux plus âgés de contenir les affects négatifs, ce qui est moins le cas pour les plus jeunes. L'utilisation intensive de la réévaluation cognitive et du coping centré sur le problème apparaît être davantage associée à des niveaux plus bas d'affects négatifs chez les plus âgés que chez les plus jeunes. Chez ces derniers, les AN restent élevés même quand ils y ont fortement recours. Deuxièmement, une relation non-linéaire en $U$, dont la courbure s'accentue avec l'âge, apparaît entre les stratégies étudiées et les affects positifs et l'activation. Ce patron peut s'expliquer dans le cadre d'une causalité réciproque reliant stratégies et affects. D'une part, dans les situations où le niveau d'affectivité positive est élevé, il n'est pas nécessaire d'avoir recours aux stratégies de régulation. 
D'autre part, si une stratégie est efficace, il est attendu que son utilisation intensive conduise également à un niveau d'affects positifs élevé, ce qui pourrait expliquer la relation en $U$ observée. Ce patron apparaît très clairement pour les plus âgés et moins chez les plus jeunes. En effet, seuls les adultes les plus jeunes relatent se trouver dans un état qui associe un faible niveau d'AP ou d'activation sans pour autant avoir recours à une des deux stratégies. Il est possible que la capacité à détecter le faible niveau d'affect positif comme déclencheur des processus de régulation tel que le mettent également en avant Congard et al. (2011) n'apparaisse qu'au cours de l'âge adulte. Le troisième résultat important concerne le coping centré sur le problème et sa relation avec la régulation des affects négatifs en fonction de l'âge. Nous observons, spécifiquement chez les personnes les plus âgées de l'échantillon, une co-occurrence d'un niveau faible d'utilisation du coping centré sur le problème et d'affectivité négative élevée, comme si cette stratégie avait un statut protecteur chez ces personnes. Chez les adultes d'âge intermédiaire ou plus jeunes, un niveau faible d'utilisation de cette stratégie coexiste avec un niveau bas d'AN, probablement dans des situations de vie favorables qui ne nécessitent pas la mise en œuvre du coping problème, comme s'ils ne l'utilisaient qu'en réponse à un événement déclenchant des affects négatifs. À l'opposé, pour les personnes plus âgées, son utilisation pourrait être de nature plus proactive, c'est-à-dire en prévention des événements négatifs afin d'empêcher la survenue des affects négatifs. Dans le même sens, Gross et Levenson (1993) suggèrent que, suivant l'âge, les personnes utilisent différemment les stratégies avec une focalisation accrue sur les antécédents avec l'avancée en âge. De plus, Blanchard-Fields (2007) explique que les âgés peuvent s'appuyer sur leur vécu socioémotionnel pour résoudre plus efficacement les problèmes auxquels ils font face. Ils pourraient gagner ainsi en proactivité.

\subsection{Conclusion}

En conclusion, et dans la continuité des modèles de la Sélection socioémotionnelle de Carstensen (1995) et du SOC-ER d'Urry et Gross (2010), nos résultats suggèrent qu'au début du développement adulte les individus auraient une utilisation exploratoire des stratégies de régulation. Chaque stratégie serait utilisée de façon équivalente et stable, se révélant parfois peu efficace notamment pour réduire les affects négatifs. Puis, les individus apprendraient les caractéristiques des stratégies, leur permettant d'en avoir une utilisation plus efficace une fois l'affect apparu. Il s'agirait alors, pour les adultes d'âge intermédiaire, d'une utilisation réactive des stratégies qui se caractériserait par une augmentation de l'utilisation de certaines stratégies, avec une préférence pour la réévaluation cognitive et 
le coping centré sur le problème. Puis, avec l'avancée en âge, il s'opérerait un changement d'objectifs visant à privilégier un état de sérénité. Les individus adopteraient alors une utilisation plus préventive des stratégies, notamment du coping centré sur le problème. Certaines stratégies seraient utilisées de façon plus fréquente et stable et deviendraient de plus en plus efficaces dans la régulation des affects. Nos résultats portent sur les effets des stratégies considérées de façon indépendantes les unes des autres, afin de mieux comprendre les évolutions de chacune. Il est cependant clair que dans la régulation, les individus utilisent de façon plus ou moins simultanée les différentes stratégies qu'ils ont à leur disposition. Il serait alors intéressant de compléter les analyses déjà réalisées afin de les compléter en considérant les interactions possibles entre les stratégies, c'est-à-dire l'utilisation conjointe de l'ensemble des stratégies.

\subsection{Limites}

Cette étude présente cependant quelques limites, parmi lesquelles l'utilisation de l'âge comme seule variable explicative de l'expertise des stratégies de régulation. La théorie de la sélection socioémotionnelle précise que c'est davantage la perception de temps futur que l'âge qui joue un rôle dans les modifications des motivations liées à l'effet de positivité, même si ces deux variables sont très liées $(\mathrm{r}=-0,70 ; \mathrm{p}<0,001$; Lang \& Carstensen, 2002). Il est possible que ce choix méthodologique ait conduit à limiter l'amplitude de l'effet de positivité observé. Il serait alors intéressant de vérifier la répétition de nos résultats avec une échelle de la perception de temps futur (Carstensen \& Lang, 1996). Concernant les stratégies de régulation, plutôt que de considérer globalement la réévaluation cognitive comme nous l'avons fait dans cette étude (à l'instar de Gross \& John, 2003), il serait pertinent de distinguer détachement et réévaluation positive. Certains travaux récents (Shiota \& Levenson, 2009) tendent effectivement à montrer que l'évolution avec l'âge de ces deux stratégies est différente : la réévaluation positive serait plus utilisée chez les personnes âgées, tandis que le recours au détachement diminuerait du fait de la baisse des fonctions exécutives avec le vieillissement. De plus, les stratégies sont ici évaluées de manière rétrospective sur l'intervalle des trois heures précédentes. Bien qu'il s'agisse d'une période relativement courte dont on peut penser qu'elle est moins soumise à la reconstruction mnésique, on ne peut exclure totalement l'existence de biais rétrospectifs (Stone, Schwarts, Neale, Shiffman, Marco, Hickcox, Paty, Porter, \& Cruise, 1998). En outre, ce patron développemental général nécessite plusieurs éléments de confirmation empirique. Par exemple, le caractère plus proactif 
de l'utilisation des stratégies chez les personnes plus âgées pourrait être investigué en prenant davantage en compte les relations temporelles entre affects et stratégies. Il serait également intéressant d'interroger le coût cognitif de l'utilisation des stratégies pour tester le phénomène d'optimisation des ressources tel que l'exprime le modèle du SOC-ER (Urry \& Gross, 2010) et l'influence que peuvent avoir les capacités d'introspection des participants sur leur fonctionnement : par exemple est-ce que les personnes âgées arrivent mieux à identifier et décrire ce qu'elles ressentent que les plus jeunes? De plus, dans ce protocole nous n'avons pas interrogé directement les participants à propos des événements de vie rencontrés. Une telle évaluation induit un coût non négligeable dans un protocole de sondage en situation de vie quotidienne et présente une validité relative car les affects ressentis orientent la perception des événements de vie. Plusieurs aspects de nos résultats, en particulier l'émergence d'un facteur général d'utilisation des stratégies, pourraient s'interpréter comme le reflet de l'effet de l'environnement sur les affects et les processus de régulation. Il nous semble que la mise au point de techniques d'identification de la variabilité intra-individuelle provoquée par les événements de vie est un des enjeux méthodologiques pour les recherches futures.

Reçu le 27 juillet 2014.

Révision acceptée le 27 avril 2015.

\section{BIBLIOGRAPHIE}

Baltes, P. B., \& Baltes, M. M. (1990). Psychological perspectives on successful aging: The model of selective optimization with compensation. In P. B. Baltes \& M. M. Baltes (Eds.), Successful aging : Perspectives from the behavioral sciences (pp. 1-34). New York: Cambridge University Press.

Bandura, A. (1999). Social cognitive theory: An agentic perspective. Asian Journal of Social Psychology, 2, 21-41.

Bates, D., Maechler, M., Bolker, B., \& Walker, S. (2013). Ime4 : Linear mixed-effects models using Eigen and S4. ( $\mathrm{R}$ package version 1.0-4) [computer software]. Retrieved from
http://CRAN.R-project.org/package=lme4 Blanchard-Fields, F. (2007). Everyday problem solving and emotion: An adult developmental perspective. Current Directions in Psychological Science, 16, 26-31.

Bolger, N., \& Laurenceau, J. (2013). Intensive longitudinal methods: An introduction to diary and experience sampling research. New York, NY US: Guilford Press.

Boujut, E, \& Bruchon-Schweitzer, M. (2007). Rôle de certains facteurs psychosociaux dans la réussite universitaire d'étudiants de première année. L'Orientation Scolaire et Professionnelle, 36, 157-177. 
Cacioppo, J. T., \& Berntson, G. G. (1994) Relationship between attitudes and evaluative space: A critical review, with emphasis on the separability of positive and negative substrates. Psychological Bulletin, 115, 401423.

Cacioppo, J. T., Hawkley, L. C., Kalil, A., Hughes, M. E., Waite, L., \& Thisted, R. A. (2008). Happiness and the invisible threads of social connection: The Chicago Health, Aging, and Social Relations Study. In M. Eid \& R. Larsen (Eds.), The science of well-being (pp. 195-219). New York: Guilford

Carstensen, L. L. (1995). Evidence for a lifespan theory of socioemotional selectivity. Current Directions in Psychological Science, 4, 151-156.

Carstensen, L. L., Gross, J. J., \& Fung, H. (1998). The social context of emotional experience. In K.W. Schaie \& M.P. Lawton (Eds.), Annual review of gerontology and geriatrics, 17, 325-352.

Carstensen, L. L., Pasupathi, M., Mayr, U., \& Nesselroade, J. R. (2000). Emotional experience in everyday life across the adult life span. Journal of personality and social psychology, 79, 644-655.

Carstensen, L. L, Turan, B., Scheibe, S., Ram, N., Ersner-Hershfield, H., Samanez-Larkin, G. R. et al. (2011). Emotional experience improves with age : evidence based on over 10 years of experience sampling. Psychology and Aging, 26, 21-33.

Charles, S. T., \& Carstensen, L. L. (2008). Unpleasant situations elicit different emotional responses in younger and older adults. Psychology and Aging, 23, 495-504.

Charles, S. T., \& Pasupathi, M. (2003). Age-related patterns of variability in selfdescriptions : implications for everyday affective experience. Psychology and Aging, 18, 524-536.

Charles, S. T., Reynolds, C. A., \& Gatz, M. (2001). Age-related differences and change in positive and negative affect over 23 years. Journal of Personality and Social Psychology, 80, 136-151.

Congard, A., Dauvier, B., Antoine, P., \& Gilles, P.-Y. (2011). Integrating personality, daily life events and emotion : Role of anxiety and positive affect in emotion regulation dynamics. Journal of Research in Personality, 45, 372-384.

Fredrickson, B. L., \& Carstensen, L. L. (1990). Choosing social partners: How old age and anticipated endings make us more selective. Psychology and Aging, 5, 335-347.

Garnefski, N., van den Kommer, T., Kraaij, V., Teerds, J., Legerstee, J., \& Onstein, E. (2002). The relationship between cognitive emotion regulation strategies and emotional problems : comparison between a clinical and a non-clinical sample. European Journal of Personality, 16, 403-420.

Gardiner, J. C., Luo, Z., \& Roman, L. A. (2009). Fixed effects, random effects and GEE: What are the differences? Statistics in Medicine, 28, 221-239.

Gross, J. J. (1998). Antecedent- and response-focused emotion regulation : Divergent consequences for experience, expression, and physiology. Journal of Personality and Social Psychology, 74, 224-237.

Gross, J. J., Carstensen, L. L., Pasupathi, M., Tsai, J., Skorpen, C. G., \& Hsu, A. Y. (1997). Emotion and aging: experience, expression, and control. Psychology and Aging, 12, 590-599.

Gross, J. J., \& John, O. P. (2003). Individual differences in two emotion regulation processes : Implications for affect, relationships, and well-being. Journal of Personality and Social Psychology, 85, 348-362.

Gross, J. J., \& Levenson, R. W. (1993). Emotional suppression: Physiology, selfreport, and expressive behavior. Journal of Personality and Social Psychology, 64, 970-986. 
Gross, J. J., \& Thompson, R. A. (2007). Emotion regulation: Conceptual foundations. In J.J. Gross (Ed.), Handbook of Emotion Regulation (pp. 3-24). New York: Guilford.

Gross, J. J., Richards, J. M., \& John, O. P. (2006). Emotion regulation in everyday life. In D. K. Snyder, J. A. Simpson, \& J. N. Hughes (Eds.), Emotion regulation in families: Pathways to dysfunction and health (pp. 13-35). Washington DC: American Psychological Association.

Guillaume, C., Eustache, F., \& Desgrange, B. (2009). L'effet de positivité: un aspect intrigant du vieillissement. Revue $\mathrm{Neu}$ ropsychologique, 1, 247-253.

Hektner, J. M., Schmidt, J. A., \& Csikszentmihalyi, M. (Eds.). (2006). Experience sampling method: Measuring the quality of everyday life. Thousand Oaks, CA: Sage Publications.

Hojsgaard, S., Halekoh, U., \& Yan, J. (2006). The R Package geepack. Generalized Estimating Equations. Journal of Statistical Software, 15, 1-11.

Kuppens, P. (2010). From appraisal to emotion. Emotion Review, 2, 157-158.

Lang, F. R. \& Carstensen, L. L. (2002). Time counts: Future time perspective, goals and social relationships. Psychology and Aging, 17, 125-139.

Larsen, J. T., McGraw, A. P., \& Cacioppo, J. T. (2001). Can people feel happy and sad at the same time? Journal of Personality and Social Psychology, 81, 684-696.

Lazarus, R. S. (1991). Emotion \& Adaptation. New York: Oxford University Press.

Lazarus, R. S., \& Folkman, S. (1984). Stress, appraisal, and coping. New York: Springer.

Luminet, O. (2002). Psychologie des émotions : Confrontation et évitement. Bruxelles : De Boeck \& Larcier.

Mroczek, D. K., \& Kolarz, C. M. (1998). The effect of age on positive and negative affect: A developmental perspective on happiness. Journal of Personality and Social Psychology, $75,1333-1349$.
Nesselroade, J. R., Gerstorf, D., Hardy, S. A., \& Ram, N. (2007). Idiographic filters for psychological constructs. Measurement, 5, 217-235.

Piolat, A., \& Bannour, R. (2008). Émotions et affects : Contribution de la psychologie cognitive. In P. Nagy \& D. Boquet (Eds.), Le sujet des émotions au Moyen Age (pp. 53-84). Paris : Beauchesne Éditeur.

Prenda, K. M., \& Lachman, M. E. (2001). Planning for the future: A life management strategy for increasing control and life satisfaction in adulthood. Psychology and Aging, 16, 206-216.

Röcke, C., Li, S.-C., \& Smith, J. (2009). Intraindividual variability in positive and negative affect over 45 days: Do older adults fluctuate less than young adults? Psychology and Aging, 24, 863-878.

Russell, J. A. (1980). A circomplex model of affect. Journal of Personality and Social Psychology, 39, 1161-1178.

Russell, J. A., \& Barrett, L. F. (1999). Core affect, prototypical emotional episodes, and other things called emotion: Dissecting the elephant. Journal of Personality and Social Psychology, 76, 805-819.

Russell, J. A., \& Carroll, J. M. (1999). On the bipolarity of positive and negative affect. Psychological Bulletin, 125, 3-30.

Scheibe, S., English, T., Tsai, J. L., \& Carstensen, L. L. (2013). Striving to feel good: ideal affect, actual affect, and their correspondence across adulthood. Psychology and Aging, 28, 160-71.

Shiota, M. N., \& Levenson, R. W. (2009). Effects of aging on experimentally instructed detached reappraisal, positive reappraisal, and emotional behavior suppression. Psychology and Aging, 24, 890-900.

Stanley, D. J., \& Meyer, J. P. (2009). Two-dimensional affective space: A new approach to orienting the axes. Emotion, 9, 214-237.

Stone, A. A., Schwartz, J. E., Neale, J. M., Shiffman, S., Marco, C. A., Hickcox, M. et 
al. (1998). How accurate are current coping assessments? A comparison of momentary versus end-of-day reports of coping efforts. Journal of Personality and Social Psychology, 74, 1670-1680.

Stone, A. A., Schwartz, J. E., Broderick, J. E., \& Deaton, A. (2010). A snapshot of the age distribution of psychological well-being in the United States. Proceedings of the National Academy of Sciences, 17, 9985-9990.

Urry, H. L., \& Gross, J. J. (2010). Emotion regulation in older age. Current Directions in Psychological Science, 19, 352-357.

Vieillard, S., \& Harm, J. (2013). La régulation des émotions au cours du vieillissement normal : revue critique. L'Année Psychologique, 113, 595-628.

Voelkle, M. C., Ebner, N. C., Lindenberger, U., \& Riediger, M. (2013). Here we go again: anticipatory and reactive mood responses to recurring unpleasant situations throughout adulthood. Emotion, 13, 424-433.

Watson, D. (1988). Intra-individual and inter-individual analyses of positive and negative affect: Their relations to health complaints, perceived stress, and daily activities. Journal of Personality and Social Psychology, 54, 1020-1030.
Watson, D., \& Clark, L. A. (1984). Negative affectivity : the disposition to experience aversive emotional states. Psychological Bulletin, 96, 465-90.

Watson, D., \& Tellegen, A. (1999). Issues in the dimensional structure of affect-Effects of descriptors, measurement error, and response formats: Comment on Russell and Carroll. Psychological Bulletin, 125, 601-610.

Wilkinson, G. N., \& Rogers, C. E. (1973). Symbolic description of factorial models for analysis of variance. Applied Statistics, 22, 392-399.

Wilt, J., Funkhouser, K., \& Revelle, W. (2011). The dynamic relationships of affective synchrony to perceptions of situations. Journal of Research in Personality, 45, 309321.

Yan, J. (2002). Geepack: Yet another package for Generalized Estimating Equations. R-News, 2, 12-14.

Yan, J. \& Fine, J. P. (2004). Estimating equations for association structures statistics. Medicine, 23, 859-880.

Yik, M., Russell, J. A., \& Steiger, J. H. (2011). A 12-point circomplex structure of core affect. Emotion, 11, 705-731. 


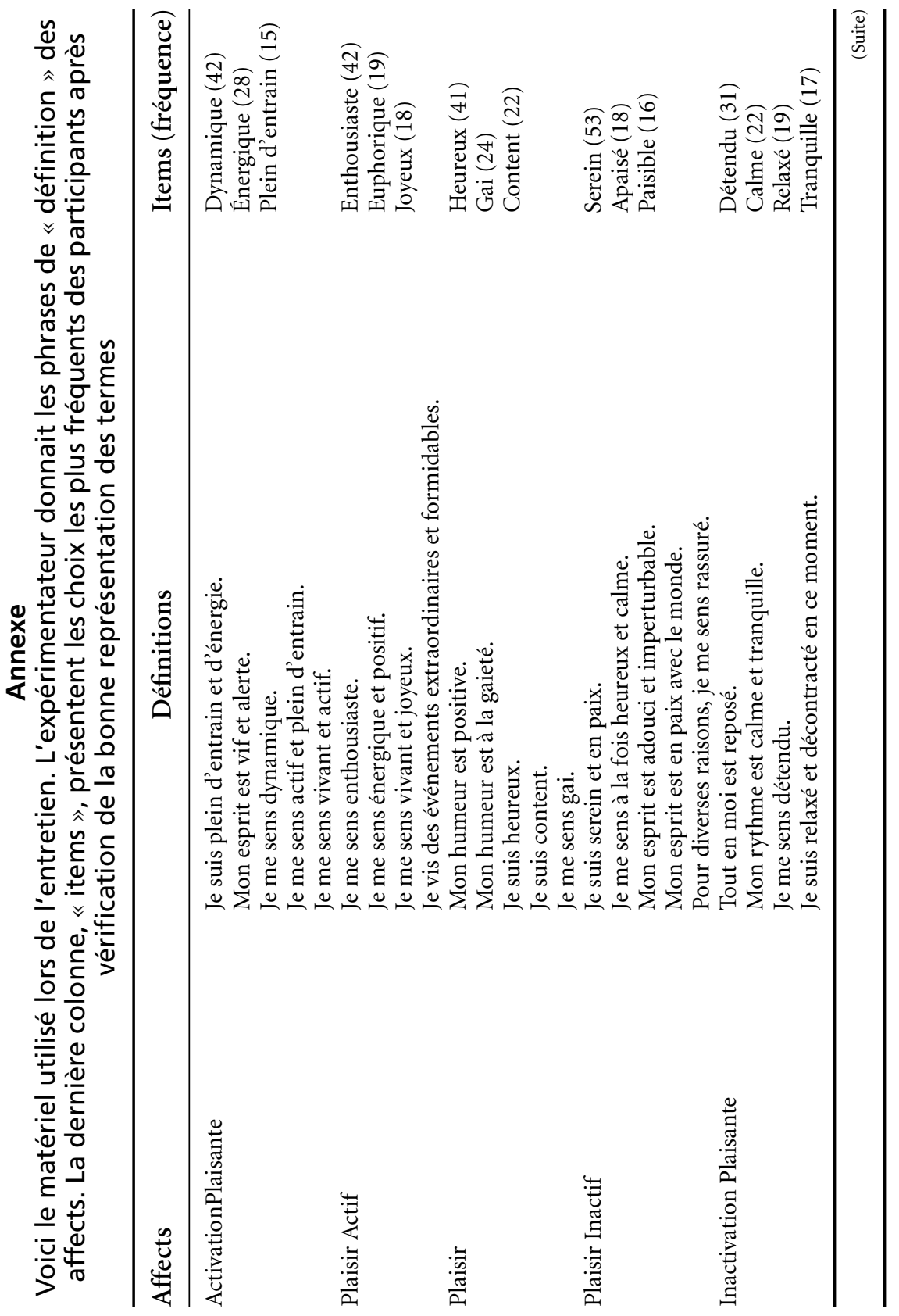




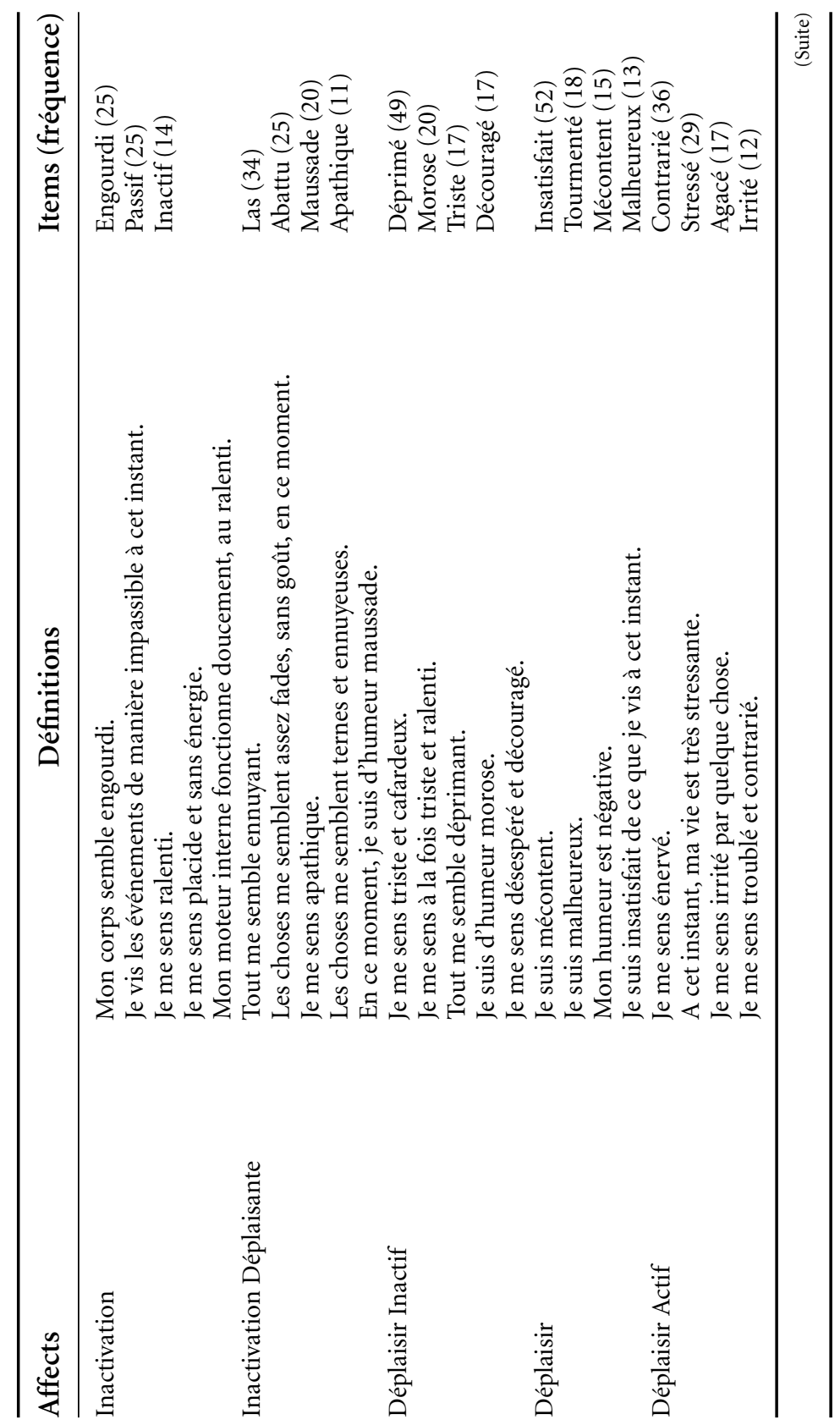




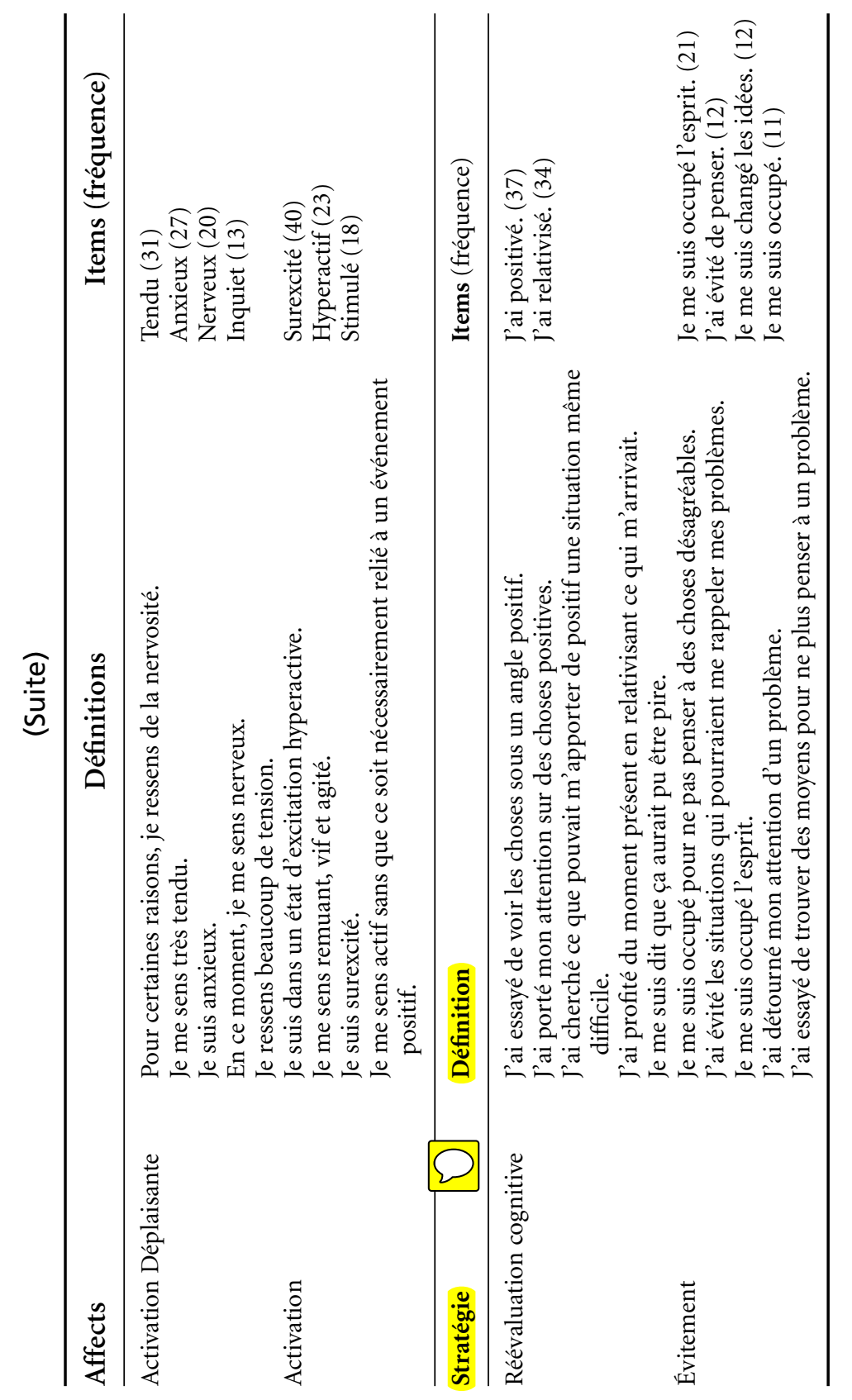




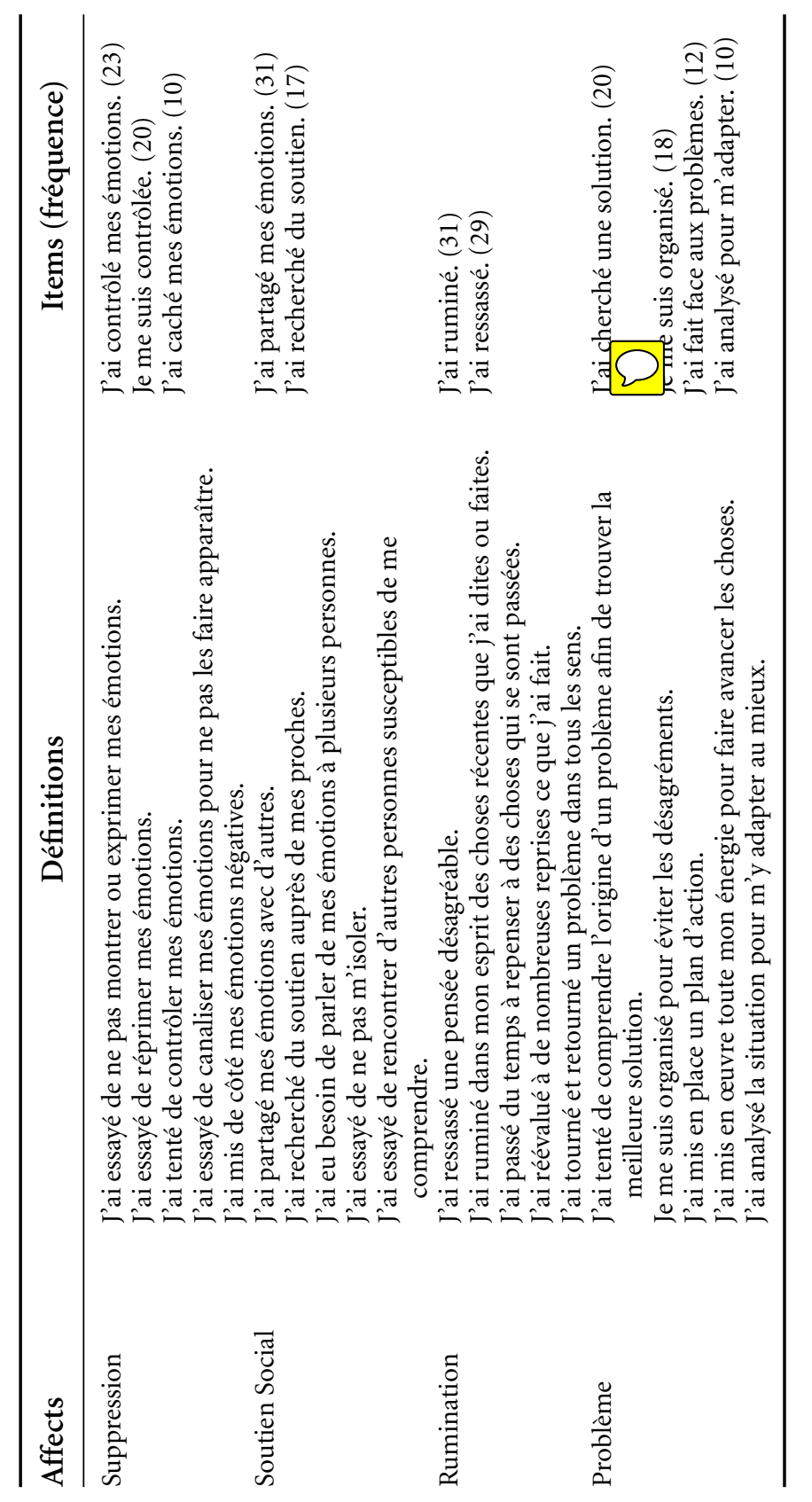




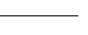

- 